\title{
Brown adipose tissue density measured by near-infrared time- resolved spectroscopy in Japanese, across a wide age range
}

Sayuri Fuse

Shinsuke Nirengi

Shiho Amagasa

Toshiyuki Homma

Ryotaro Kime

Tasuki Endo

Naoki Sakane

Mami Matsushita

Masayuki Saito

Takeshi Yoneshiro

Yuko Kurosawa

Takafumi Hamaoka 


\title{
Brown adipose tissue density measured by near-infrared time-resolved spectroscopy in Japanese, across a wide age range
}

\author{
Sayuri Fuse,${ }^{a, \dagger}$ Shinsuke Nirengi ${ }^{\mathrm{b}, \dagger}$ Shiho Amagasa, ${ }^{\mathrm{c}}$ Toshiyuki Homma, ${ }^{\mathrm{d}}$ Ryotaro Kime, ${ }^{\mathrm{a}}$ Tasuki Endo, ${ }^{\mathrm{a}}$ \\ Naoki Sakane, ${ }^{\mathrm{b}}$ Mami Matsushita, ${ }^{\mathrm{e}}$ Masayuki Saito, ${ }^{\mathrm{f}}$ Takeshi Yoneshiro, ${ }^{\mathrm{g}}$ Yuko Kurosawa, ${ }^{\mathrm{a}}$ and \\ Takafumi Hamaoka a,*

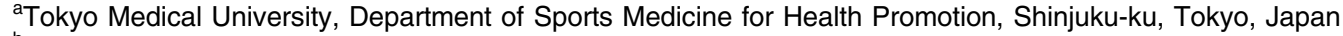 \\ ${ }^{b}$ Clinical Research Institute, National Hospital Organization Kyoto Medical Center, Division of Preventive Medicine, Fukakusa, Kyoto, Japan \\ 'Tokyo Medical University, Department of Preventive Medicine and Public Health, Shinjuku-ku, Tokyo, Japan \\ dDaito Bunka University, Faculty of Sports and Health Science, Higashimatsuyama-shi, Saitama, Japan \\ eTenshi College, Department of Nutrition, Higashi-ku, Sapporo, Japan \\ ${ }^{f}$ Hokkaido University, Kita-ku, Sapporo, Japan \\ gUniversity of California, UCSF Diabetes Center, Department of Cell and Tissue Biology, San Francisco, California, United States
}

\begin{abstract}
F}$-fluorodeoxyglucose (FDG)-positron emission tomography (PET) along with computed tomography (CT) is a standard method for assessing brown adipose tissue (BAT) activity. We tested the usefulness of near-infrared time-resolved spectroscopy $\left(\mathrm{NIR}_{\mathrm{TRS}}\right)$ as a simple and noninvasive method for evaluating BAT density (BAT-d) by examining the effects of some factors known to influence BAT activity. The total hemoglobin concentration as a parameter of BAT-d was evaluated using NIR $\mathrm{R}_{\text {TRS }}$ in the supraclavicular region in 413 Japanese individuals. The associations were analyzed between BAT-d and sex, age, the percentages of body fat (\%BF), visceral fat (VF), and the seasonal ambient temperature (AmT) fluctuations. Age was associated with decreased BAT-d $(P<0.05)$. There was no sex difference in the BAT-d, except for those in their twenties. Multivariate analyses revealed that \%BF and VF were correlated with BAT-d, and the lower AmT (around $4^{\circ} \mathrm{C}$ or $5^{\circ} \mathrm{C}$ ) for 4 and 6 weeks prior to the measurement day was associated with an increase in the BAT-d. Our NIR TRS results were analogous to those reported with ${ }^{18} \mathrm{FDG}-\mathrm{PET} / \mathrm{CT}$, indicating the usefulness of NIR $\mathrm{TRS}_{\text {. BAT-d might }}$ increase during the 4 and 6 weeks after the AmT decreases to lower than $4^{\circ} \mathrm{C}$ or $5^{\circ} \mathrm{C}$. $\odot$ The Authors. Published by SPIE under a Creative Commons Attribution 3.0 Unported License. Distribution or reproduction of this work in whole or in part requires full attribution of the original publication, including its DOI. [DOI: 10.1117/1.JBO.23.6.065002]
\end{abstract}

Keywords: near-infrared spectroscopy; noninvasive, brown adipose tissue; age; ambient temperature.

Paper 180021RR received Jan. 12, 2018; accepted for publication May 21, 2018; published online Jun. 13, 2018.

\section{Introduction}

Active brown adipose tissue (BAT) has been detected by ${ }^{18} \mathrm{~F}$ fluorodeoxyglucose (FDG)-positron emission tomography (PET) combined with computed tomography (CT) ${ }^{1-4}$ in adults and neonates, as well as animals. It has been reported that human BAT activity is related to cold-induced thermogenesis, ${ }^{5}$ body weight, ${ }^{5}$ and glucose tolerance.${ }^{6,7}$ Furthermore, daily cold exposure increased the BAT activity in healthy individuals, ${ }^{5,8,9}$ obese individuals, ${ }^{10}$ and patients with type 2 diabetes. ${ }^{7}$ Thus, BAT is expected to be a potential tool to combat obesity and lifestyle-related diseases.

Most epidemiological studies related to BAT have been informed by work in oncology and used ${ }^{18}$ FDG-PET/CT in thermoneutral conditions. These data showed that BAT activity was higher in women, younger individuals, and lean patients, as well as under conditions of lower ambient temperatures (AmT). ${ }^{11-17}$ However, since these data were collected for the diagnosis of cancer, data on healthy adults were lacking, and the mean age was biased toward patients older than 60 years *Address all correspondence to: Takafumi Hamaoka, E-mail: kyp02504@ nifty
.com

TThe authors contributed equally to this work. of age. In addition, the measurements were conducted under thermoneutral conditions, wherein the FDG uptake might have been underestimated. ${ }^{1,18}$ Indeed, the ${ }^{18}$ FDG-PET/CT-positive BAT ratio is variable; in studies involving cancer patients without cold exposure, the ratio was found to be extremely low $(\sim 5 \%){ }^{11-17,19,20}$ However, in younger healthy individuals, who were exposed to cold, the ratio was much higher $(\sim 50 \%$ to $100 \%))^{3,6}$ With regards to the effect of AmT on the activation of BAT, the results are conflicting with one study demonstrating that the rise in the BAT activity was delayed by a few months in association with a beginning in the decline in the $\mathrm{AmT}^{11}$ and the other study stating that BAT activity was higher in early winter than late winter or early spring. ${ }^{21}$

Matsushita et al. ${ }^{6}$ and Yoneshiro et al. ${ }^{22}$ showed that functional BAT could be epidemiologically evaluated using cold-stimulated ${ }^{18}$ FDG-PET/CT, in relatively healthy younger adults. They demonstrated that BAT activity was also negatively correlated with age, body fatness, and glucose metabolism. However, there were no sex differences in the BAT activity; ${ }^{6,22}$ this was inconsistent with results of the aforementioned oncology studies, in which experimental cold exposure was not utilized. Thus, the difference in the sensitivity, between men and women, to mild outdoor cold exposure might be prominent in oncology studies. 
In general, the near-infrared time-resolved spectroscopy $\left(\mathrm{NIR}_{\mathrm{TRS}}\right)$ method can evaluate the total hemoglobin concentration [total-Hb], which is an index of the blood volume or tissue vasculature. Therefore, this method can be used for vascular density evaluations, wherein BAT is expected to exhibit a higher density than that exhibited by white adipose tissue. Recently, we demonstrated, in humans, that the [total- $\mathrm{Hb}$ ], determined by $\mathrm{NIR}_{\mathrm{TRS}}$, under thermoneutral conditions, is positively correlated with cold-induced ${ }^{18}$ FDG-PET/CT parameters, only in the supraclavicular region, which potentially contains BAT deposits. $^{23}$ As we found no difference between the [total-Hb] at $27^{\circ} \mathrm{C}$ and at the end of a 2-h-long exposure to cold conditions at $19^{\circ} \mathrm{C}$, we were able to measure the [total- $\mathrm{Hb}$ ] for the estimation of the vascularity of BAT, using $\mathrm{NIR}_{\mathrm{TRS}}$, without conditions of cold exposure. Collectively, the $\mathrm{NIR}_{\mathrm{TRS}}$ method is expected to be suitable for evaluating BAT density (BAT-d) in men and women, which is equivalent to the active BAT intensity or the amount of BAT, as determined by ${ }^{18}$ FDG-PET/CT with cold exposure. ${ }^{23-26}$ As the $\mathrm{NIR}_{\mathrm{TRS}}$ method was introduced only in 2015, there are no data on the relationship of BAT with age, sex, anthropometrical parameters, and environmental factors. To verify the usefulness of $\mathrm{NIR}_{\mathrm{TRS}}$, there is a need to accumulate evidence on analogous data with regards to ${ }^{18}$ FDG-PET/CT with cold exposure. It is also important to obtain new evidence on human BAT-d, through epidemiological studies, using $\mathrm{NIR}_{\mathrm{TRS}}$, as otherwise, ${ }^{18}$ FDG-PET/CT measurement would be impossible, owing to several limitations such as the lack of accessibility to the relevant device and radiation exposure.

Thus, the purpose of this cross-sectional study was to confirm the relationship between BAT-d, determined through the use of $\mathrm{NIR}_{\mathrm{TRS}}$, and anthropometrical parameters, such as body fatness, to determine the differences in BAT-d, in terms of age and sex. We also aimed to identify influencing factors, such as seasonal fluctuations in the AmT on BAT-d, in Japanese individuals, across a wide age range.

\section{Methods}

Volunteers were recruited through advertisements on posters or direct contact in three different areas-Hokkaido, Shiga Prefecture, and Tokyo, Japan. After the participants arrived at the laboratory, the following parameters were measured: BAT-d, height, body weight, percentage of body fat (\%BF), visceral fat area (VFA), subcutaneous fat thickness in the supraclavicular region, systolic blood pressure (SBP), diastolic blood pressure (DBP), and heart rate (HR). The room temperature was regulated from $23^{\circ} \mathrm{C}$ to $27^{\circ} \mathrm{C}$ by the use of an air conditioner and heater. In addition, data on the AmT and day length, in each region, were obtained from the Japan Meteorological Agency ${ }^{27}$ and the National Astronomical Observatory of Japan, ${ }^{28}$ respectively. The study design and protocols were approved by the institutional review boards of Ritsumeikan University and Tokyo Medical University, in accordance with the ethical principles contained in the Declaration of Helsinki. Written informed consent was obtained from all the participants. These studies were conducted in the cold weather season (December, January, February, and March) from 2013 to 2017.

\subsection{Participants}

The 504 participants recruited for this study were aged over 20 years. Most of them were physically active, at a recreational level, but those who participated in regular athletic sports training more than two days per week $(n=34)$ were excluded.
Three participants were excluded because of an intake of capsinoids, which are reported to activate and recruit BAT. ${ }^{5,24}$ In addition, 54 participants were excluded because the VFA in 16 of them was under $10 \mathrm{~cm}^{2}$, which was a measurement limitation of using bioelectrical impedance analysis (EW-FA90; Panasonic, Osaka, Japan); the remaining 38 did not agree to undergo VFA measurements. Finally, we analyzed 413 volunteers (185 men and 228 women), aged 20 to 85 years (Table 1 ).

\subsection{Measurement of Anthropometric and Circulatory Parameters}

Body mass index (BMI) was calculated as follows: body weight in kilograms divided by the square of the height in meters $\left(\mathrm{kg} / \mathrm{m}^{2}\right)$. We calculated the body surface area using the DuBois equation: body surface area $\left(\mathrm{m}^{2}\right)=$ height $(\mathrm{cm})^{0.725} \times$ body weight $(\mathrm{kg}) 0.425 \times 71.84 \times 10^{-4}$. The $\% \mathrm{BF}$ was estimated by a dual-energy $\mathrm{x}$-ray absorptiometry scan (Lunar Prodigy; GE Healthcare, Buckinghamshire, United Kingdom) or the multifrequency bioelectric impedance method (Inbody 720 Body Composition Analyzer; Biospace, Seoul, Republic of Korea). The VFA, at the abdominal level of L4-L5, was estimated using 1.5-T magnetic resonance imaging (Signa HDxt; GE Healthcare, Buckinghamshire, United Kingdom) or a bioelectrical impedance analysis (EW-FA90; Panasonic, Osaka, Japan). The subcutaneous fat thickness at supraclavicular region was measured by B-mode ultrasonography (Vscan Dual Probe; GE Vingmed Ultrasound AS, Horten, Norway). The subcutaneous fat thickness was measured by the investigator using the attached distance measuring system and calculated as the mean value for three points (Fig. 1). SBP, DBP, and HR were measured by an automated sphygmomanometer (HBP-9020; Omron Healthcare, Kyoto, Japan).

\subsection{Brown Adipose Tissue Density Measurements}

The BAT-d, evaluated by the [total-Hb] using NIR TRS $_{\text {(TRS-20; }}$ Hamamatsu Photonics K.K., Hamamatsu, Japan), was measured for $1 \mathrm{~min}$ at $23^{\circ} \mathrm{C}$ to $27^{\circ} \mathrm{C}$. The probes were placed on the skin of the supraclavicular region that potentially contained BAT, and participants were required to remain in a sitting position during the measurements, as previously described. ${ }^{23-26}$ Compared with visible light wavelengths, NIR wavelengths (700 to $3000 \mathrm{~nm}$ ) show lesser scattering and, consequently, better penetration into biological tissue. However, light absorption by water limits tissue penetration above the $900-\mathrm{nm}$ wavelength; thus, the 650 - to 900-nm range is suitable for measurements. ${ }^{29}$ Accordingly, we used NIR wavelengths of 760,800 , and $830 \mathrm{~nm}$ to evaluate oxy-hemoglobin concentration, deoxy-hemoglobin concentration, and [total-Hb]. With the $3-\mathrm{cm}$ probe used in this study, light can reach a mean depth of $2 \mathrm{~cm},{ }^{30}$ where BAT is potentially located. ${ }^{31}$

The tissue was illuminated using a $200-\mu \mathrm{m}$-core-diameter optical fiber by the pulsed light generated from picoseconds light pulses, with 100-ps full width at half-maximum, a 5-MHz repetition rate, and an average power of $80 \mu \mathrm{W}$ of each wavelength. The emitted photons penetrated the tissue and were reflected to a 3-mm-diameter optical bundle fiber, through which they were sent to a photomultiplier tube for single-photon detection and a signal processing circuit for time-resolved measurement. Using the nonlinear least-squares method, the digitized temporal profile data from an in-vitro sample or tissue were fitted with a theoretical temporal profile, derived from the 


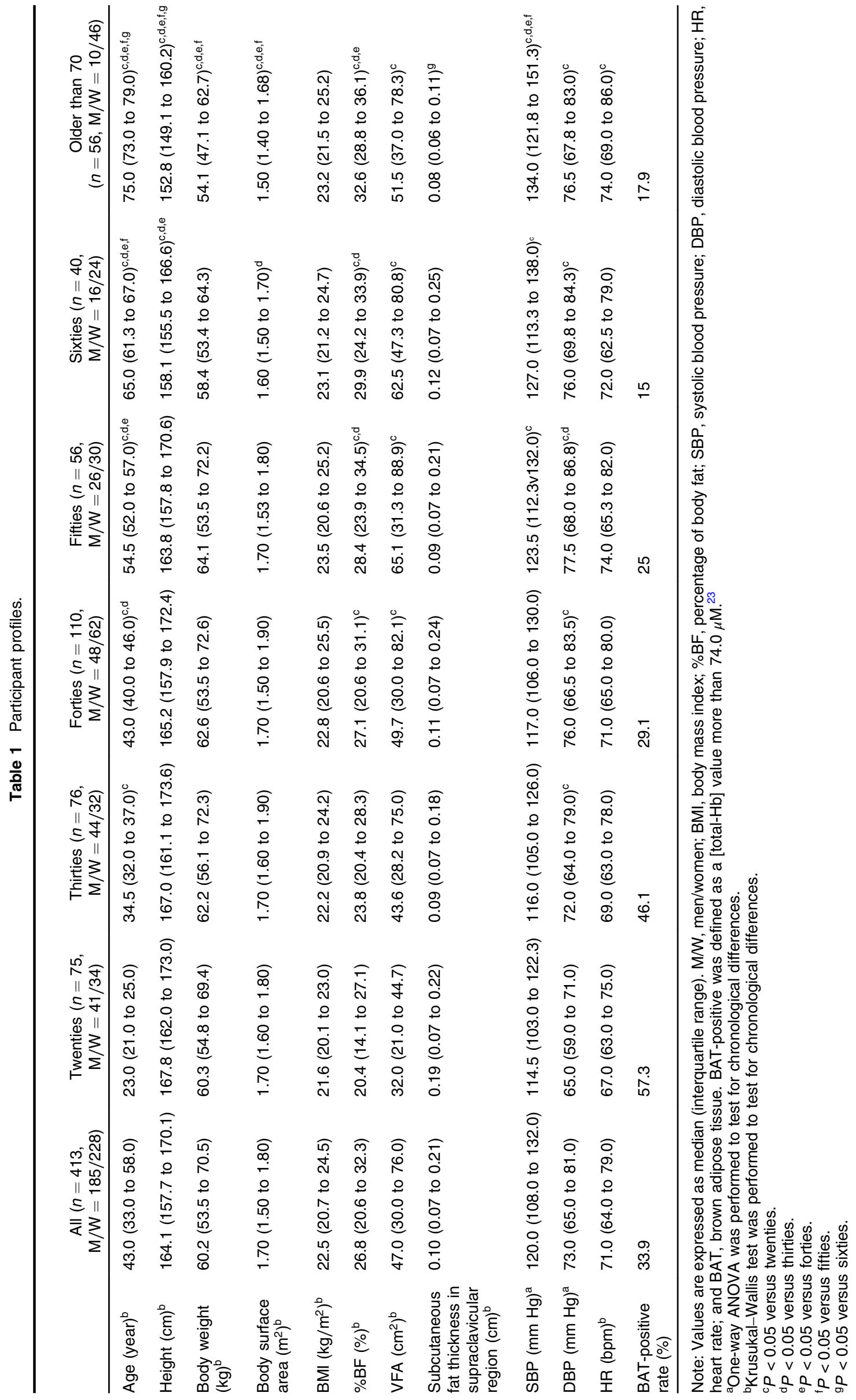




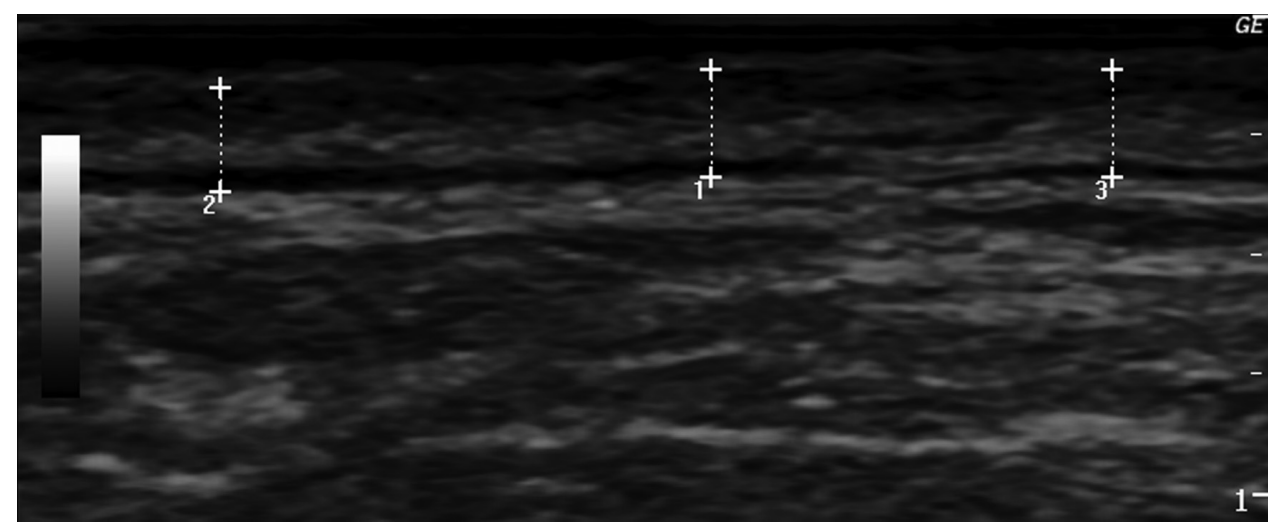

Fig. 1 A typical image of the subcutaneous fat layer in the supraclavicular region This is an ultrasonic image of the supraclavicular region in a 27-year-old man. The subcutaneous fat layer was measured using B-mode ultrasonography (Vscan Dual Probe; GE Vingmed Ultrasound AS, Horte, Norway), whereas the thickness was measured using the attached distance measuring system by an investigator and calculated as the mean value for three points. The mean thickness in this region is $0.26 \mathrm{~cm}$.

analytical solution of the photon diffusion theory with a semiinfinite homogeneous reflectance model. After convolution with the instrumental response function, the time response of the instrument itself could be compensated for, and the absorption coefficient values and reduced scattering coefficient values, at 760, 800, and $830 \mathrm{~nm}$, were obtained using the least-squares fitting method. Then, the absolute [total-Hb] was calculated as the sum of oxy-hemoglobin and deoxy-hemoglobin concentrations. ${ }^{23,29}$ The NIR $\mathrm{TRS}$ system collected data every $10 \mathrm{~s}$. The coefficient of variation for repeated measurements of the [total-Hb] was $4.9 \% .^{23}$

Our previous study indicated that a cutoff value of $74.0 \mu \mathrm{m}$ [total-Hb] for distinguishing BAT negativity from BAT positivity (evaluated by FDG-PET/CT) resulted in the best prediction accuracy of $82.8 \%$, with a sensitivity of $75.0 \%$, specificity of $100 \%$, positive predictive value of $100 \%$, and negative predictive value of $64.3 \% .^{23}$

\subsection{Average Ambient Temperature During the Day and Day Length}

To examine the effect of AmT and day length on BAT-d, data on the average AmT during the day and day length were obtained from the Japan Meteorological Agency ${ }^{27}$ and the National Astronomical Observatory of Japan, ${ }^{28}$ respectively. We collected data from the area nearest to where each participant livedHokkaido, Shiga Prefecture, or Tokyo. We searched for data on the day of measurement and 2, 4, and 6 weeks prior to the measurement day. We also calculated the average AmT and day length during the 2 and 4 weeks before, 4 and 6 weeks before, and 2, 4, and 6 weeks before the measurement day.

\subsection{Statistical Analyses}

Data are expressed as either mean \pm standard error (SE) or median with interquartile range. If normality was detected by the Shapiro-Wilk test, we used one-way analysis of variance (ANOVA) to test for chronological differences in the anthropometric and circulatory parameters, with post hoc testing using the Tukey's test. If normality was not detected, the Krusukal-Wallis test was conducted, and then, the Bonferroni's test was used if there was a significant difference. The Jonckheere-Terpstra test was conducted to reveal the trend of the chronological changes in the BAT-positive ratio. Correlations were assessed using Kendall's correlation coefficient. The independent associations of age, sex, and BAT-d with body fatness were estimated using stepwise multiple regressions; in a similar manner, age, sex, AmT, and day length, as predictors of BAT-d, were tested. To conduct the analyses, the categorical variables were set at " 0 " for women and " 1 " for men. The correlation coefficient between the nominal scale and ratio scale was shown as the correlation ratio. Following the stepwise multivariate regression analysis of the independent associations of age, sex, AmT, and day length with BAT-d, analysis of covariance was conducted to investigate the relationship between BAT-d and AmT, adjusting for \%BF and VFA. The range of the AmT was divided into fourths and then further divided equally. A two-way ANOVA was used to test the interactions (sex $\times$ chronological group) and main effects (sex and chronological group), adjusting for BMI, \%BF, and VFA. If there was a significant interaction or main effect, the sex or chronological differences in the variables were analyzed using Bonferroni's test. All analyses were performed using SPSS (IBM SPSS Statistics 24, IBM Japan, Tokyo, Japan) and $P<0.05$ was considered statistically significant.

\section{Results}

The [total-Hb] in the supraclavicular region was measured in a total of 413 participants using NIR $_{\mathrm{TRS}}$, during the cold weather season, in Japan. The participants had a median age of 43.0 (33.0 to 58.0 ) years, BMI of $22.5(20.7$ to 24.5$) \mathrm{kg} / \mathrm{m}^{2}$, and $\%$ BF of $26.8 \%$ (20.6\% to $32.3 \%$ ) (Table 1). As shown in Table 1, the BAT-positive ratio was $33.9 \%$ in all the participants (140/413), and it tended to significantly decrease with age; it was more than $50 \%$ in those in their twenties (43/75) but less than $20 \%$ in those in their sixties (6/40) and those older than 70 years $(10 / 56)$.

To assess the impact of BAT-d, age, and sex on body fatness, we conducted univariate and multivariate analyses. The univariate regression analysis showed that BAT-d, age, and sex significantly was correlated with BMI, \%BF, and VFA, respectively (Table 2). The multivariate regression analysis revealed that BAT-d and sex were determinants of BMI, \%BF, and VFA, but age did not affect BMI (Table 2). 
Table 2 Independent associations of age, sex, and BATs with body fatness.

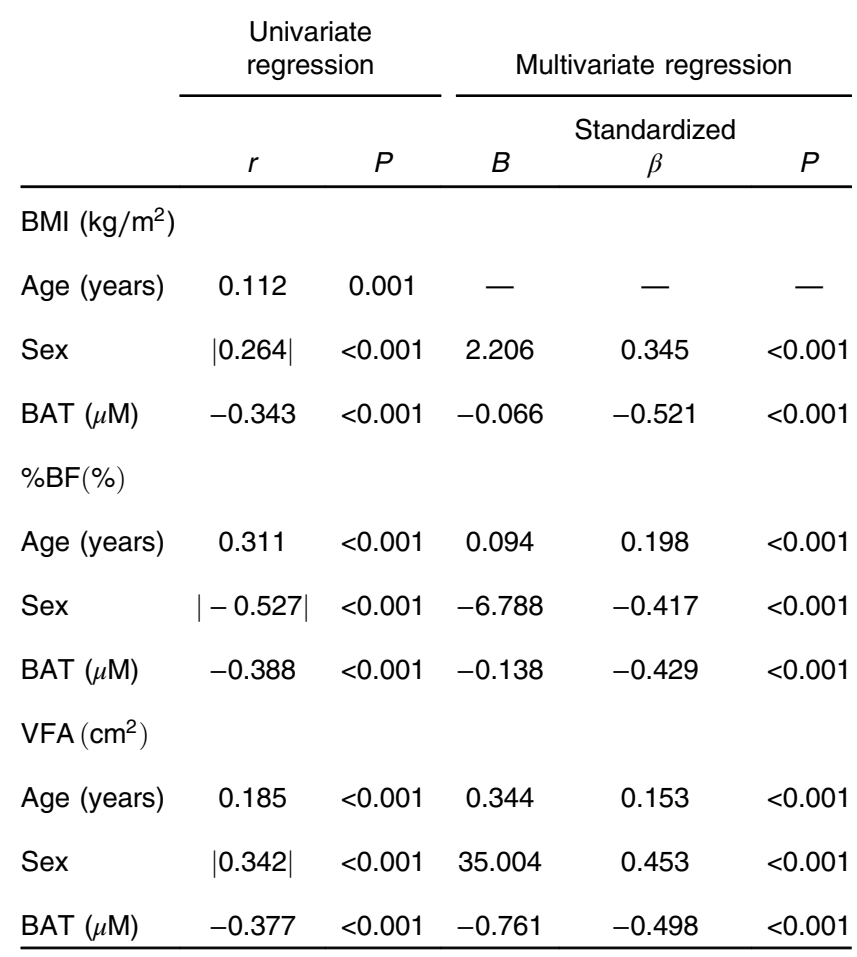

Note: BMI, body mass index; \%BF, percent body fat; and BAT, brown adipose tissue. The categorical variables were set at "0" for women and "1" for men, respectively. The correlation coefficient between the nominal scale and the ratio scale was shown as the correlation ratio.

After controlling for BMI, \%BF, and VFA, we observed chronological and sex differences in the BAT-d. As shown in Fig. 2, the BAT-d of participants who were in their sixties was significantly lower than that of those in their twenties. A significant interaction (sex $\times$ chronological group) was noted, as was the presence of a significant main effect in the chronological group but not in terms of sex (Fig. 2). A significant sex difference was observed only among those in their twenties
(Fig. 2). The BAT-d of participants older than 50 years was significantly decreased compared to that of men in their twenties (Fig. 2).

To assess the factors influencing BAT-d, univariate and multivariate regression analyses were conducted. The univariate analysis showed sex, age, the mean AmT 6 weeks before the measurement day, and the average AmT during the 4 and 6 weeks before to be significant determinants of BAT-d; however, the multivariate regression analysis revealed that age and the average AmT during the 4 and 6 weeks before the measurement day remained determinants of BAT-d (Table 3). Although some of the AmT indices were significantly correlated with BAT, there was no significant correlation between BAT and day length (Table 3).

As a significant result, following multivariate regression analysis, we investigated the relation between BAT-d and the average AmT during the 4 and 6 weeks before the measurement day after controlling for $\% \mathrm{BF}$ and VFA. The BAT-d, when measured at temperatures lower than $3.7^{\circ} \mathrm{C}$ of the average AmT, during the 4 and 6 weeks before the measurement day, was significantly higher than that measured at temperatures higher than $5.0^{\circ} \mathrm{C}$ (Fig. 3).

\section{Discussion}

In the present cross-sectional study, we applied $\mathrm{NIR}_{\mathrm{TRS}}$ to evaluate the BAT-d in 413 Japanese individuals, aged 20 to 85 years. Our study has three main findings. First, we confirmed that BAT-d was negatively correlated with body fatness. Second, older age was associated with decreased BAT-d, with positive activity rates of BAT observed in those in their fifties and sixties, and those above 70 -years old at $25.0 \%, 15.0 \%$, and $17.9 \%$, respectively. Third, the average ambient lower temperature, during the 4 and 6 weeks prior to the measurement day, under free-living situations, was associated with an elevation in the BAT-d. In particular, the [total-Hb], at an average AmT lower than $3.7^{\circ} \mathrm{C}$, during the 4 and 6 weeks prior to the measurement day, was higher than that observed at temperatures higher than $5.0^{\circ} \mathrm{C}$.

The positive rate of BAT observed in our study (33.9\%), as determined in the same manner as the $\mathrm{NIR}_{\mathrm{TRS}}$ measurements, ${ }^{23}$ was comparable or slightly lower than that obtained in a

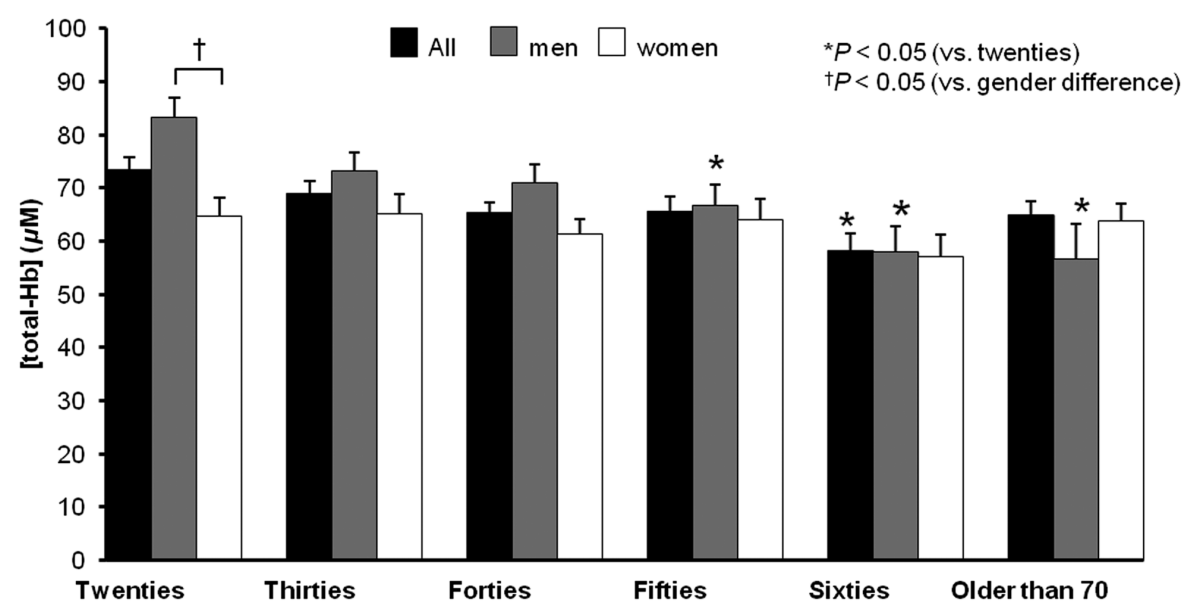

Fig. 2 Chronological and sex differences in terms of BAT. The [total- $\mathrm{Hb}]$ in the supraclavicular region potentially contains BAT. The values are expressed as means \pm SE, adjusting for BMI, body fat ratio, and VFA. 
Table 3 Independent associations of age, sex, outdoor temperature, and day length with BAT.

\begin{tabular}{|c|c|c|c|c|c|}
\hline \multirow[b]{2}{*}{ BAT } & \multicolumn{2}{|c|}{$\begin{array}{l}\text { Univariate } \\
\text { regression }\end{array}$} & \multicolumn{3}{|c|}{ Multivariate regression } \\
\hline & $r$ & $P$ & $B$ & Standardized $\beta$ & $P$ \\
\hline Age (years) & -0.252 & $<0.001$ & -0.508 & -0.345 & $<0.001$ \\
\hline Sex & $|0.155|$ & 0.002 & - & - & - \\
\hline Mean outdoor temperature on measurement day $\left({ }^{\circ} \mathrm{C}\right)$ & -0.035 & 0.302 & - & - & - \\
\hline Mean outdoor temperature two weeks prior to measurement day $\left({ }^{\circ} \mathrm{C}\right)$ & 0.039 & 0.245 & - & - & - \\
\hline Mean outdoor temperature four weeks prior to measurement day $\left({ }^{\circ} \mathrm{C}\right)$ & -0.045 & 0.174 & - & - & - \\
\hline Mean outdoor temperature six weeks prior to measurement day $\left({ }^{\circ} \mathrm{C}\right)$ & -0.082 & 0.014 & - & - & - \\
\hline Average temperature between two and four weeks before $\left({ }^{\circ} \mathrm{C}\right)$ & -0.008 & 0.806 & - & - & - \\
\hline Average temperature between four and six weeks before $\left({ }^{\circ} \mathrm{C}\right)$ & -0.078 & 0.019 & -1.022 & -0.145 & 0.002 \\
\hline Average temperature between two, four, and six weeks before $\left({ }^{\circ} \mathrm{C}\right)$ & -0.029 & 0.389 & - & - & - \\
\hline Day length on measurement day (h) & 0.023 & 0.494 & - & - & - \\
\hline Day length two weeks prior to measurement day (h) & 0.032 & 0.336 & - & - & - \\
\hline Day length four weeks prior to measurement day (h) & 0.042 & 0.269 & - & - & - \\
\hline Day length six weeks prior to measurement day $(h)$ & 0.049 & 0.145 & - & - & - \\
\hline Average day length between two and four weeks before (h) & 0.036 & 0.276 & - & - & - \\
\hline Average day length between four and six weeks before $(h)$ & 0.050 & 0.130 & - & - & - \\
\hline Average day length between two, four, and six weeks before (h) & 0.039 & 0.239 & - & - & - \\
\hline
\end{tabular}

Note: BAT, brown adipose tissue. The categorical variables were set at "0" for women and "1" for men, respectively. The correlation coefficient between the nominal scale and the ratio scale was shown as the correlation ratio.

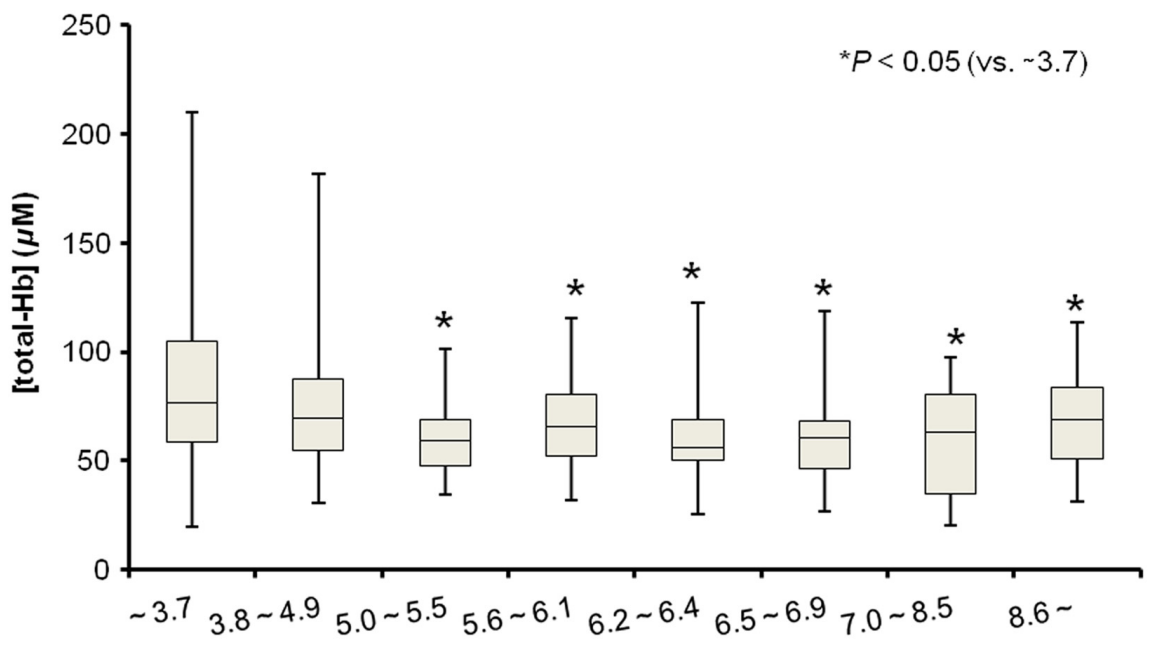

The average temperature at four and six weeks before the measurement day $\left({ }^{\circ} \mathrm{C}\right)$

Fig. 3 The relation between BAT and the average temperature at four and six weeks before the measurement day. The [total- $\mathrm{Hb}]$ in the supraclavicular region potentially contains BAT. Analysis of covariance was conducted, adjusting for body fat ratio and VFA.

Japanese study, which used ${ }^{18}$ FDG-PET/CT with cold exposure $(41.4 \%){ }^{22}$ The reason for the relatively lower rate could be the fact that our study included elderly adults, with a median age of 43.0 years, whereas the previously conducted study predominantly included those in the age range of 20 to 40 years (median age of 26.0 years). ${ }^{6}$ In fact, the positive rates of BAT observed in the participants in their twenties (57.3\% versus $53.0 \%)$, thirties (46.1\% versus $39.5 \%$ ), and forties $(29.1 \%$ versus $26.9 \%)$ were similar to those noted in another study. ${ }^{22}$ The finding of the positive rate of BAT among those in their fifties (25.0\%), sixties 
$(15.0 \%)$, and adults over the age of 70 years $(17.9 \%)$ is new, in Japanese settings.

We showed that body fatness parameters were negatively correlated with BAT-d, which is consistent with the results of various studies that used ${ }^{18} \mathrm{FDG}-\mathrm{PET} / \mathrm{CT}$, regardless of the presence of cold exposure. ${ }^{6,14-17,19,20}$ Although we should consider the possibility that the BAT-d was affected by subcutaneous fat thickness, ${ }^{26}$ the subcutaneous fat thickness in the supraclavicular region is within a very narrow range and has little effect on the measured parameters. For instance, the fat thickness in the supraclavicular region ranged from 0.6 to $2.5 \mathrm{~mm}$ when evaluated by B-mode ultrasonography in this study. This corresponds to an attenuation of the NIR signal by $<10 \%$ of the assumed $0-\mathrm{mm}$ fat layer thickness. ${ }^{32}$ After the elimination of the effect of confounder factors, using stepwise multiple regressions, the relationship between BAT-d and $\mathrm{BMI}, \% \mathrm{BF}$, and VFA was obtained.

Findings on the sex differences in terms of BAT are controversial. ${ }^{6,13-17,19,21}$ BAT activity was found to be higher in women, in many oncology studies. ${ }^{13-17,20}$ However, no sex differences were found in ${ }^{18} \mathrm{FDG}$-PET/CT studies with cold exposure. ${ }^{6,22}$ The oncology studies had very large sample sizes but did not include cold exposure, whereas the studies using ${ }^{18}$ FDG-PET/CT with cold exposure had relatively small sample sizes. In this study, we evaluated BAT-d in 413 individuals, using $\mathrm{NIR}_{\mathrm{TRS}}$, which is equivalent to ${ }^{18} \mathrm{FDG}-\mathrm{PET} / \mathrm{CT}$ with cold exposure. As a result, the fact that BAT-d is higher in women was not confirmed. Further studies are necessary to assess sex differences in terms of BAT.

This is the first study to demonstrate that the average ambient lower temperature, during the 4 and 6 weeks prior to the measurement day, under free-living situations, is associated with an elevation in the BAT-d. Laboratory studies showed that daily cold exposure $\left(10^{\circ} \mathrm{C}\right.$ to $17^{\circ} \mathrm{C}, 2$ to $6 \mathrm{~h} /$ day $)$, when dressed in unnaturally light clothing (t-shirt with underwear), for 10 days to 6 weeks, promoted BAT recruitment, ${ }^{5,8-10}$ and the results of these previous studies might provide a hint for the consideration of our result. Cohade et al. ${ }^{11}$ showed a rise in the monthly prevalence of BAT activity during the winter, but the rise was delayed by a few months, in relation to the beginning of a decrease in the AmT. Kim et al. ${ }^{21}$ showed that BAT activity was higher in early winter than later winter or early spring. The temperature at which some cold exposure studies were carried out at can be equivalent to a degree in our cold season. However, since the type of clothing could have varied and the duration of the daily cold exposure in unknown, it is difficult to compare the results of our study with those of other studies. Despite the undetermined duration of the daily cold exposure needed to increase functional BAT activity/mass, in this study, we found that the [total-Hb], at an average AmT lower than $3.7^{\circ} \mathrm{C}$, during the 4 and 6 weeks prior to the measurement day, was higher than that at temperatures higher than $5.0^{\circ} \mathrm{C}$. This observation suggests that BAT-d increases during the 4 and 6 weeks after the average AmT drops to $<4^{\circ} \mathrm{C}$ or $5^{\circ} \mathrm{C}$. A larger variation in the [total- $\mathrm{Hb}$ ], at a lower temperature, indicates differences in the individual sensitivity to the exposure to cold environments; participants with a low BAT-d could be insensitive while differing sensitivities could have been present among those with a high BAT-d. Because we could not acquire relevant longitudinal data, we are uncertain of the effects of an increase in the outside temperature on BAT-d. However, according to a thermogenic capsinoid supplementation study conducted to increase BAT-d, ${ }^{24}$ the cessation of supplementation during an increase in the outside temperature decreased BAT-d. Therefore, we speculate that BAT-d would gradually decrease several months later, when the outside temperature increases during spring. Further studies, with a longitudinal design and larger sample size, are needed, along with the monitoring of the degree and duration of daily cold environments, to determine the critical AmT cutoff value needed to recruit functional BAT under free-living situations.

There was no significant correlation between BAT density and day length, in this study. Contrary to our results, one previously conducted study showed, in its univariate analysis, that a shorter day length was positively correlated with BAT activity. ${ }^{12}$ However, that study did not include cold exposure before ${ }^{18}$ FDG-PET/CT measurements; ${ }^{12}$ therefore, the AmT, which is related to day length, on the measurement day, may be confounded. Although Ouellet et al. ${ }^{15}$ also demonstrated, through an univariate analyses, the presence of a significant relationship between day length and BAT activity, the relationship was not significant in the multivariate analyses.

The validity of evaluating BAT-d using $\mathrm{NIR}_{\mathrm{TRS}}$ has been verified in previously conducted studies. ${ }^{23,24}$ We reported a significant relationship between BAT-d, as evaluated by the [total-Hb] using $\mathrm{NIR}_{\mathrm{TRS}}$, and BAT activity, as evaluated by ${ }^{18}$ FDG-PET/CT. We have already shown that, in younger individuals, the [total-Hb] can be increased, and that the degree of the increase in BAT-d and BAT activity is almost similar (48.8\% and $46.4 \%$, respectively) after daily thermogenic capsinoid ingestion, ${ }^{24}$ which is known to increase BAT-d and/or mass. We also found that the [total-Hb] tended to decrease during the 8-week follow-up period, after capsinoid treatment. ${ }^{24}$

Precise determination of the volume of interest is difficult using the NIR spectroscopic (NIRS) method. On the basis of a continuous wave NIRS study in the milk model by Chance et $\mathrm{al}^{33}$ if we assume the volume of interest to be a rotation body of the ellipse revolving across $180 \mathrm{deg}$ or a hemisphere with a 3-cm optode separation, it can be estimated to be $\sim 4 \mathrm{~cm}^{3}$. However, as a banana-like pattern has been reported for photon migration, ${ }^{34}$ the above assumption might be inappropriate. Moreover, according to a recent study, the mean depth of the light penetration with a $3-\mathrm{cm}$ optode separation using NIR $_{\mathrm{TRS}}$ would be $\sim 2 \mathrm{~cm},{ }^{30}$ which is greater than half of the emitterdetector separation reported in many previous studies. . $^{2,33,34}$ Although $\mathrm{NIR}_{\mathrm{TRS}}$ is noninvasive, uncomplicated, less expensive than PET/CT, and free of radiation exposure, for the evaluation of tissue oxygenation, in humans, ${ }^{23}$ further studies on the volume measured using NIR $_{\mathrm{TRS}}$ are needed.

Although several studies have reported an increase in the BAT activity in younger individuals, the question of whether BAT activity can be increased in individuals with a lower BAT-d and/or advancing age remains unresolved. ${ }^{35-37}$ In this study, we found that even some individuals over their fifties had BAT-positive rates of $15 \%$ to $25 \%$, and this suggests the possibility to maintain and/or increase the BAT-d even in relatively older individuals. However, the effect of genetic predispositions cannot be ruled out for individuals with a higher BAT-d.

This study has several limitations. It included a relatively small number of participants, although some other studies on BAT also involved only a small sample size. The presence of selection bias and variation in the age distribution cannot be ruled out. The cross-sectional design has the inherent disadvantage of defining causality. We did not evaluate BAT activity using 
the well-established, standardized ${ }^{18}$ FDG-PET/CT method, although several studies have validated the use of NIR $\mathrm{TRS}_{\text {. }}$

In conclusion, the results of this cross-sectional study showed that a decrease in BAT-d is associated with advancing age and the lower AmT was associated with an increase in the BAT-d through the use of $\mathrm{NIR}_{\mathrm{TRS}}$, in a wide age range of participants. Our results are consistent with those of previously conducted ${ }^{18} \mathrm{FDG}-\mathrm{PET} / \mathrm{CT}$ studies. In particular, the average AmT, which was lower than $4^{\circ} \mathrm{C}$ or $5^{\circ} \mathrm{C}$ during the 4 and 6 weeks prior to the measurement day, might be a determining factor for increasing BAT-d. As the study sample of this cross-sectional and exploratory study was too small to draw a conclusion, further studies are needed to identify the factors that affect BAT-d, which take into consideration genetic predisposition and environmental factors, such as clothing, physical activity levels, and nutritional factors.

\section{Disclosures}

The authors declared no conflicts of interest.

\section{Acknowledgments}

This work was supported by JSPS KAKENHI Grant Number 15H03100. The authors wish to acknowledge the volunteers who participated in this study.

\section{References}

1. M. Saito et al., "High incidence of metabolically active brown adipose tissue in healthy adult humans: effects of cold exposure and adiposity," Diabetes 58(7), 1526-1531 (2009).

2. A. M. Cypess et al., "Identification and importance of brown adipose tissue in adult humans," N. Engl. J. Med. 360(15), 1509-1517 (2009).

3. W. D. van Marken Lichtenbelt et al., "Cold-activated brown adipose tissue in healthy men," N. Engl. J. Med. 360(15), 1500-1508 (2009).

4. K. A. Virtanen et al., "Functional brown adipose tissue in healthy adults," N. Engl. J. Med. 360(15), 1518-1525 (2009).

5. T. Yoneshiro et al., "Recruited brown adipose tissue as an antiobesity agent in humans," J. Clin. Invest. 123(8), 3404-3408 (2013).

6. M. Matsushita et al., "Impact of brown adipose tissue on body fatness and glucose metabolism in healthy humans," Int. J. Obes. (London) 38(6), 812-817 (2014).

7. M. J. Hanssen et al., "Short-term cold acclimation improves insulin sensitivity in patients with type 2 diabetes mellitus," Nat. Med. 21(8), 863-865 (2015).

8. D. P. Blondin et al., "Increased brown adipose tissue oxidative capacity in cold-acclimated humans," J. Clin. Endocrinol. Metab. 99(3), E438E446 (2014).

9. A. A. van der Lans et al., "Cold acclimation recruits human brown fat and increases nonshivering thermogenesis," J. Clin. Invest. 123(8), 3395-3403 (2013).

10. M. J. Hanssen et al., "Short-term cold acclimation recruits brown adipose tissue in obese humans," Diabetes 65(5), 1179-1189 (2016).

11. C. Cohade, K. A. Mourtzikos, and R. L. Wahl, “USA-Fat': prevalence is related to ambient outdoor temperature-evaluation with 18F-FDG PET/CT," J. Nucl. Med. 44(8), 1267-1270 (2003).

12. I. T. Au-Yong et al., "Brown adipose tissue and seasonal variation in humans," Diabetes 58(11), 2583-2587 (2009).

13. P. Lee et al., "A critical appraisal of the prevalence and metabolic significance of brown adipose tissue in adult humans," Am. J. Physiol. Endocrinol. Metab. 299(4), E601-E606 (2010).

14. C. Pfannenberg et al., "Impact of age on the relationships of brown adipose tissue with sex and adiposity in humans," Diabetes 59(7), 1789-1793 (2010).

15. V. Ouellet et al., "Outdoor temperature, age, sex, body mass index, and diabetic status determine the prevalence, mass, and glucose-uptake activity of ${ }^{18}$ F-FDG-detected BAT in humans," J. Clin. Endocrinol. Metab. 96(1), 192-199 (2011).
16. H. A. Jacene et al., "The relationship between patients' serum glucose levels and metabolically active brown adipose tissue detected by PET/CT," Mol. Imaging. Biol. 13(6), 1278-1283 (2011).

17. A. Persichetti et al., "Prevalence, mass, and glucose-uptake activity of ${ }^{18}$ F-FDG-detected brown adipose tissue in humans living in a temperate zone of Italy," PLoS One 8(5), e63391 (2013).

18. C. A. Garcia et al., "Reduction of brown fat 2-deoxy-2-[F-18]fluoro-Dglucose uptake by controlling environmental temperature prior to positron emission tomography scan," Mol. Imaging Biol. 8(1), 24-29 (2006).

19. X. Shao et al., "Characterization of brown adipose tissue ${ }^{18}$ F-FDG uptake in PET/CT imaging and its influencing factors in the Chinese population," Nucl. Med. Biol. 43(1), 7-11 (2016).

20. Q. Wang et al., "Brown adipose tissue activation is inversely related to central obesity and metabolic parameters in adult human," PLoS One 10(4), e0123795 (2015).

21. S. Kim et al., "Temporal relation between temperature change and FDG uptake in brown adipose tissue," Eur. J. Nucl. Med. Mol. Imaging 35(5), 984-989 (2008).

22. T. Yoneshiro et al., "Age-related decrease in cold-activated brown adipose tissue and accumulation of body fat in healthy humans," Obesity (Silver Spring) 19(9), 1755-1760 (2011).

23. S. Nirengi et al., "Human brown adipose tissue assessed by simple noninvasive near-infrared time-resolved spectroscopy," Obesity (Silver Spring) 23(5), 973-980 (2015).

24. S. Nirengi et al., "Assessment of human brown adipose tissue density during daily ingestion of thermogenic capsinoids using near-infrared time-resolved spectroscopy," J. Biomed. Opt. 21(9), 091305 (2016).

25. S. Nirengi et al., "Daily ingestion of catechin-rich beverage increases brown adipose tissue density and decreases extramyocellular lipids in healthy young women," Springerplus 5(1), 1363 (2016).

26. S. Nirengi et al., "Seasonal differences in brown adipose tissue density and pulse rate variability in a thermoneutral environment," J. Physiol. Anthropol. 37, 6 (2018).

27. Japan Meteorogical Agency, "Weather observations," http://www.jma .go.jp/jma/index.html (29 May 2018).

28. National Astronomical Observatory of Japan, “Local calendar," https:// www.nao.ac.jp/en/ (29 May 2018).

29. T. Hamaoka et al., "Near-infrared spectroscopy/imaging for monitoring muscle oxygenation and oxidative metabolism in healthy and diseased humans," J. Biomed. Opt. 12(6), 062105 (2007).

30. S. Gunadi et al., "Spatial sensitivity and penetration depth of three cerebral oxygenation monitors," Biomed. Opt. Express 5(9), 2896-2912 (2014).

31. A. Flynn et al., "Contrast-enhanced ultrasound: a novel noninvasive, nonionizing method for the detection of brown adipose tissue in humans," J. Am. Soc. Echocardiogr. 28(10), 1247-1254 (2015).

32. M. Niwayama, "Quantitative measurement of muscle hemoglobin oxygenation using near-infrared spectroscopy with correction for the influence of a subcutaneous fat layer," Rev. Sci. Instrum. 71, 4571 (2000).

33. B. Chance et al., "Comparison of time-resolved and -unresolved measurements of deoxyhemoglobin in brain," Proc. Natl. Acad. Sci. U. S. A. 85, 4971-4975 (1988).

34. W. Cui, C. Kumar, and B. Chance, "Experimental study of migration depth for the photons measured at sample surface. I. Time resolved spectroscopy and imaging," Proc. Int. Soc. Opt. Eng. 1431, 180-191 (1991).

35. E. P. Broeders et al., "The bile acid chenodeoxycholic acid increases human brown adipose tissue activity," Cell Metab. 22(3), 418-426 (2015).

36. A. L. Carey et al., "Ephedrine activates brown adipose tissue in lean but not obese humans," Diabetologia 56(1), 147-155 (2013).

37. A. M. Cypess et al., "Activation of human brown adipose tissue by a $\beta 3$-adrenergic receptor agonist," Cell Metab. 21(1), 33-38 (2015).

Sayuri Fuse is an appointed assistant professor in the Department of Sports Medicine for Health Promotion, Tokyo Medical University. She received her $\mathrm{PhD}$ in medicine from Tokyo Medical University in 2017. Her research interests include exercise physiology and brown adipose tissue (BAT).

Shinsuke Nirengi is a postdoctoral researcher at the National Hospital Organization Kyoto Medical Center. He received his PhD 
in sport and health science from the Ritsumeikan University in 2015. His research interests include BAT, exercise physiology, and biomedical optics.

Shiho Amagasa is a PhD student at Tokyo Medical University. Her research interests include physical activity and sedentary behavior, exercise epidemiology, and lifestyle-related disease prevention.

Toshiyuki Homma is an associate professor at the Faculty of Sports and Health Science, Daito Bunka University. He has received a Young Investigators Award, Eighth Congress of European College of Sports Science in 2003. He received his PhD from Tokyo Medical University in 2005. His specialization is exercise physiology. His current major research interest is effective training for sports performance and health from a perspective of muscle energy metabolism.

Ryotaro Kime is a senior lecturer at Tokyo Medical University. He has conducted research on muscle oxidative metabolism using nearinfrared and phosphorus-magnetic resonance spectroscopies with Professor Britton Chance. He has received research awards, such as a Young Investigators Award, Fourth Congress of European College of Sport Science in 1999. His research expertise is exercise physiology and control of muscle oxidative metabolism.

Tasuki Endo is a PhD student at Tokyo Medical University. His research interests include exercise physioloty and biomedical optics.

Naoki Sakane received his MD degree from Jichi Medical School in 1989. He received $\mathrm{PhD}$ in medicine from Kyoto Prefectural University of Medicine in 1999. Currently, he is working as a division director at Division of Preventive Medicine, Clinical Research Institute, National Hospital Organization. His research interests include brown adipose tissue, beta3-adrenoceptor polymorphism, and beta3-adrenergic agonists, as well as diabetes prevention and diabetes education.
Mami Matsushita received her PhD in nutrition from Tenshi College, Japan, in 2015. She is an assistant professor and registered dietitian in the Department of Nutrition, School of Nursing and Nutrition Tenshi College. Her research interests include BAT and obesity-related diseases prevention.

Masayuki Saito received his $\mathrm{PhD}$ in biochemistry from Osaka University, Japan, in 1970. His main research field is the patho-physiology of energy metabolism and obesity, with special references to BAT. Currently, as an emeritus professor of Hokkaido University, he is working on human BAT, particularly focusing on some food ingredients activating BAT and reducing body fatness.

Takeshi Yoneshiro is a postdoctoral fellow in the Department of Cell and Tissue Biology, UCSF Diabetes Center, University of California. His research interests include the control of energy expenditure and adiposity with special reference to metabolic function of BAT in humans.

Yuko Kurosawa is a senior lecturer at Tokyo Medical University. She has received a postdoctoral award at the Cincinnati Translational Neuroscience Symposium 2007 and Best Poster Award of the International Creatine-2015 Conference. Her research expertise is creatine metabolism in brain and skeletal muscles.

Takafumi Hamaoka has conducted research on muscle oxidative metabolism using near-infrared and phosphorus-magnetic resonance spectroscopies with Professor Britton Chance. He has received research awards, such as a Young Investigators Award, First Congress of European College of Sports Science in 1996. His research expertise is exercise medicine, control of muscle oxidative metabolism, and evaluation of human adipose tissue. 